\title{
Quaderni
}

QUADERNI Communication, technologies, pouvoir

79 | Automne 2012

Produire la démocratie

\section{Les chaînes publiques et privées en Europe après la Seconde Guerre mondiale}

\section{Catherine Ghosn}

\section{(2) OpenEdition}

\section{Journals}

Édition électronique

URL : http://journals.openedition.org/quaderni/629

DOI : 10.4000/quaderni.629

ISSN : 2105-2956

\section{Éditeur}

Les éditions de la Maison des sciences de l'Homme

\section{Édition imprimée}

Date de publication : 5 octobre 2012

Pagination : 109-118

\section{Référence électronique}

Catherine Ghosn, "Les chaînes publiques et privées en Europe après la Seconde Guerre mondiale 》, Quaderni [En ligne], 79 | Automne 2012, mis en ligne le 05 octobre 2014, consulté le 20 avril 2019.

URL : http://journals.openedition.org/quaderni/629; DOI : 10.4000/quaderni.629 


\section{Communication}

\section{Les chaînes}

publiques et privées en Europe après la Après la Seconde Guerre mondiale, le projet de mettre en œuvre une action culturelle européenne grandit afin de bâtir l'Europe sur des consolidations solides. Le Conseil de l'Europe, fondé en 1949, est connu comme une des premières institutions œuvrant pour la construction européenne. En février 1950, est ratifiée l'Union européenne de radiodiffusion (UER) qui se définit comme un outil au service d'une « radiodiffusion unie en Europe » et qui vise à une communauté européenne de la télévision (Alvès, 2008) se basant sur le principe du service public.

\section{Seconde} Guerre mondiale

Une littérature conséquente en Sciences de l'information et de la communication, en Histoire, en Sociologie a rendu compte des évolutions des télévisions européennes. Nous nous sommes appuyé sur certains des ces travaux pour mener une étude comparative permettant de traduire les tendances politiques alors en œuvre et les raisons qui ont progressivement installé les chaînes privées dans le paysage audiovisuel dominé par le service public, sur le plan européen. Ces résultats permettent ensuite de mieux évaluer la place occupée par la France dans la politique de service public/privé qu'elle choisit de mener.

\section{Catherine Ghosn}

\author{
Laboratoire d'Études et
Recherches Appliquées
nces Sociales (LERASS) \\ Laboratoire d'Études et
de Recherches Appliquées
ciences Sociales (LERASS) \\ Laboratoire d'Études et
de Recherches Appliquées
en Sciences Sociales (LERASS)
}

Le service public dans une perspective européenne

L'Allemagne et la Belgique représentent deux pays assez particuliers au sein de l'Europe des douze en raison de l'importance du régime fédéraliste et de leur histoire culturelle. Si on la compare à d'autres pays européens, la télévision allemande a traversé des périodes différentes qui ont exercé sur les responsables de la radiodiffusion une influence très forte, influence d'autant 
plus importante qu'elle est née dans le contexte hitlérien. Pendant la guerre, l'Allemagne a subi les attaques massives qui ont détruit une grande partie des installations, des émetteurs et des stations. À la fin de la guerre, les Alliés ont repris en main le système de radiodiffusion pour installer un paysage audiovisuel qui ressemble plus à ce qu'ils voulaient d'une part, qui fasse table rase de la « dénazification (désintoxication des esprits) » (Defrance, 2008) pour y installer un fonctionnement plus démocratique basé sur le principe de pluralisme, d'autre part. La seconde différence essentielle consiste dans le découpage de l'Allemagne en régions (Länder), principe organique même du pays.

Le pays hérite en 1949 des structures et de la politique audiovisuelle léguée par les Alliés. Le principe de la libre information de l'opinion, surtout celui concernant la liberté de radiodiffusion, est placé sous le principe des droits fondamentaux. Les médias bénéficient de la protection de la Constitution et se caractérisent comme les principaux vecteurs de la formation des citoyens. Les principes essentiels de la radiodiffusion en Allemagne de l'Ouest ont ainsi été principalement définis en fonction du passé politique du pays (régime dictatorial et reprise en main par les alliés occidentaux). Les autres principes se basent sur le pluralisme et sur le fédéralisme pour empêcher toute possibilité d'instrumentalisation par le pouvoir en place (Chaniac, 2008). Le domaine culturel est aussi privilégié, considéré comme prioritaire sur le domaine économique. En 1950, les différents établissements de droit public fusionnent en une communauté de travail, appelée ARD (Arbeitsgemeinschaft der öffentlich rechtlichen Rundfunkanstalten der Bundesrepu- blik Deutschland) ou encore Première chaîne (Defrance, op.cit.) et à partir de 1954, la première chaîne de l'ARD émet un programme destiné à tous les citoyens allemands. Malgré des tentatives de contrôle par des hommes au pouvoir, ou encore de créations de chaînes commerciales, la signature pour la mise en place de la deuxième chaîne publique date de 1960 : la ZDF (Zweites Deutsches Fernsehen) commence à fonctionner en 1963, suivie dans la foulée par les chaînes généralistes régionales, appelées Dritte Programme retransmises dans de nombreux Länder.

Ces particularités restent spécifiques à l'Allemagne et on ne peut les appliquer dans leur globalité à d'autres pays. Seule la notion de fédéralisme peut se retrouver dans le cas de la Belgique qui compte à elle seule plusieurs particularités (Lits, 2008). Effectivement, elle :

- se caractérise comme un Ètat fédéral, dépendant d'un pouvoir fédéral,

- se compose « d'entités régionales (Flandre, Wallonie, Bruxelles) et communautaires (francophone, néerlandophone et germanophone) », - est équipée de câbles depuis longtemps et dans la majorité des foyers, permettant ainsi la réception de nombreuses chaînes étrangères,

- dépend, sur le plan de la politique audiovisuelle, de deux organismes publics communautaires, la RTBF destinée au public francophone, et la VRT au public flamand.

C'est en 1953 que deux chaînes de télévision publique naissent, l'une francophone, l'autre néerlandophone, avec des principes de programmation différents : la première opte pour la diffusion des programmes français alors issus de la RTF ; pour la seconde, les responsables du 
service audiovisuel flamand préfèrent attribuer à la télévision des valeurs qui développent les principes culturels et éducatifs liés à l'histoire de la communauté flamande, ainsi que des informations traitant de leur actualité. Les chaînes belges obéissent à la configuration suivante : elles correspondent à des critères linguistiques (francophones, néerlandophones, germanophones), appartiennent au service public ou privé, et sont identifiées comme des chaînes nationales/ communautaires ou locales, voire étrangères (Lits, op.cit.).

Si la politique télévisuelle de l'Espagne et de l'Italie ne repose pas sur le même type de découpage territorial, elle relève néanmoins d'un accord sur un découpage d'ordre politique en fonction duquel les chaînes sont attribuées à des partis.

«La télévision publique espagnole est née avec le handicap de l'absence d'un financement public. Une seconde originalité découle de l'importance prise par les régions, dotées d'un statut d'autonomie depuis la Constitution de 1978, ce qui débouchera sur la création, au cours des années 1980. de chaînes dites autonomiques, dans un contexte d'opposition politique au pouvoir central $»^{1}$.

Ces chaînes « autonomiques » ne remettent pas encore en cause le monopole du service public ; la loi de 1988 les y aide dans la mesure où elle signifie la fin du monopole public, marqué par l'arrivée de trois chaines commerciales (Canal Plus, Antena 3 et Tele 5) détenues par des opérateurs privés. Les « télévisions régionales » (Regourd, 1992) directement issues du contexte politique en référence aux campagnes électorales durant lesquelles TV3 (chaîne catalane) et ETB (chaîne basque) sont créées, et qualifiées de « télévisions d'opposition ».

En Italie, la loi de 1975 procède à une reconfiguration de la télévision publique, surtout pour ce qui concerne l'expression des différentes sensibilités politiques et la mise en œuvre du pluralisme. Ainsi la RAI ne doit pas se faire l'écho des principes du pouvoir exécutif et favoriser à l'antenne l'expression des différents courants politiques italiens (Regourd, op. cit.). Le principe de la lottizzazione (partage de la télévision publique entre les principaux partis politiques) a ainsi été progressivement mis en œuvre pour apparaître plus clairement dans les années 90 avec la manière dont les partis politiques se sont départagés les chaînes. La première chaîne de la $R A I$ se trouve sous le contrôle de la Démocratie chrétienne, la deuxième sous le couvert socialiste, et la troisième sous la main mise communiste (Regourd, op. cit.). La télévision privée s'installe en Italie par le biais des télévisions locales au cours des années 70 et l'exemple de Silvio Berlusconi dans le domaine de la communication audiovisuelle illustre bien les possibilités données au financement privé. Il met en place le réseau privé Canal 5 à partir d'une télévision locale et à partir de 1979, rachète Italia 1 et Rete 4, soit les réseaux concurrents, qui deviennent ainsi les chaînes du groupe Fininvest en concurrence avec les chaînes publiques de la $R A I$.

Ces quatre premiers pays se distinguent relativement bien par leurs différences historiques et culturelles. Il faut cependant souligner qu'ils ont été en partie influencés par le modèle de télévision britannique, considéré comme pionnier dans 
l'histoire de la télévision de service public.

Créée en 1936 dans le cadre du service public, la télévision britannique doit d'abord se plier aux réglementations qui régissent la radio nationale, tout en gardant la volonté de renforcer le modèle de service public audiovisuel considéré comme propre à la culture britannique (Aldridge, Mazdon 2008). La British Broadcasting Company (BBC) émet via le service des Postes et Télécommunications et relève du critère de « monopole d'Etat », dépendant d'un point de vue financier d'une redevance annuelle payée par les propriétaires de postes radio. Le concept de « service public» est privilégié au Royaume-Uni, tout d'abord en raison du rôle exercé par le gouvernement sur son développement (il l'a appuyé et soutenu), alors qu'il adoptait plutôt une attitude défiante à ses débuts. Le principe même de service public est aussi activé de manière significative par John Reith, premier directeur général de la $B B C$ et convaincu qu'elle doit tendre à des objectifs détachés de tout intérêt financier et servir le public (Aldridge, Mazdon, op. cit.). Cette conception assez particulière s'impose d'ailleurs dans le paysage audiovisuel européen en construction. C'est au début des années 1950 que des projets d'élargissement du paysage audiovisuel sous forme de chaînes privées commencent à naître et se précisent avec l'initiative menée par Norman Collins. Ce dernier crée la Popular Television Alliance (PTA) avant de demander l'appui d'hommes politiques et industriels. Pour les industriels qui le soutiennent, l'alternative proposée à la télévision britannique permet de diminuer dans une moindre mesure le monopole exercée par la $B B C$ et d'offrir un autre type de programmation, donc plus de choix, aux téléspectateurs (Aldridge, Mazdon op. cit). Le point d'orgue est atteint le 2 juin 1953, lors de la retransmission du couronnement de la reine Elizabeth II qui rassemble pour la première fois plus de téléspectateurs que d'auditeurs. Cet événement permet aux tenants de la télévision privée de critiquer de manière virulente le monopole détenu par la télévision publique pour la diffusion d'un moment aussi historique que celui-là. La Television Act est ainsi décrétée en 1954, laquelle permet la création d'une autre chaîne financée par des fonds privés et par la publicité, ITV (Independant Television).

Quelques éléments permettent d'étudier les points communs entre les télévisions publiques qui se trouvent dans l'espace européen après la Seconde Guerre mondiale. Dans un premier temps, le tableau ci-dessous synthétise les informations en affichant les trois principales entrées qui caractérisent les télévisions de service public des cinq pays retenus dans les parties précédentes. Les critères qui reviennent le plus souvent sont ceux de l'autonomie, du pluralisme et du fédéralisme.

Fig. 1 : Principes de la télévision de service public. Situation des pays après la Seconde Guerre mondiale.

\begin{tabular}{|c|c|c|c|}
\hline Ville & $\begin{array}{l}\text { Autonomie (relative) } \\
\text { vis-à-vis du pouvoir } \\
\text { politique }\end{array}$ & Pluralisme & $\begin{array}{l}\text { Fédéralisme- } \\
\text { principe com- } \\
\text { munautaire }\end{array}$ \\
\hline Allemagne & $\checkmark$ & $\checkmark$ & $\checkmark$ \\
\hline Belgique & & $\checkmark$ & $\checkmark$ \\
\hline Espagne & $\checkmark$ & $\checkmark$ & \\
\hline Italie & $\checkmark$ & $\checkmark$ & \\
\hline Royaume-Uni & $\checkmark$ & $\checkmark$ & \\
\hline
\end{tabular}

Dans un second temps, nous procédons à une 
analyse de ces résultats en mettant en évidence les différences et les changements impliqués par la télévision commerciale.

Des chercheurs restent plus critiques sur cette indépendance et la relativisent, ou la démentent. La $B B C$ est issue du parti au pouvoir, et c'est le Conseil des gouverneurs, nommés par la reine, qui désigne le conseil de direction de la $B B C$. Le Conseil des gouverneurs a ainsi autorité en la matière pour nommer le directeur général de la $B B C$ et les membres du conseil de direction (Regourd, op. cit.). La même dépendance politique caractérise l'autorité publique de contrôle de la télévision, IBA (Independant Broadcasting Authority) dont les douze membres sont directement nommés par le ministre de l'Intérieur. Ce dernier dispose d'une autorité directe qui lui permet d'exprimer des reproches ou des recommandations aussi bien aux membres de l'IBA que du conseil de direction de la $B B C$, et ce, au nom du gouvernement (Regourd, op . cit.). Au final, la différence entre la chaîne de service public et la chaîne privée semble beaucoup moins importante que ce qui était envisageable. ITV ne dépasse pas les limites du «populisme », tandis qu'il est encore reproché à la $B B C$ de verser dans les programmes de moindre qualité dans le seul but de toucher un audimat plus important.

Les différences des télévisions de service public en Europe viennent surtout du montant du financement, principalement assuré par la redevance. L'Angleterre et l'Allemagne fixent une redevance largement plus élevée que la France et l'Italie, alors que, paradoxalement, il n'existe pas de redevance en Espagne (Chaniac, op. cit.). Le cas de l'Espagne s'explique par le contexte historique et politique du régime franquiste pour lequel la liberté d'expression est exclue de son principe. La même logique s'applique au principe de la communication télévisuelle dont le financement est alors assuré par la publicité. Une autre différence très particulière entre ces pays réside dans le principe communautaire relatif à la Belgique et à l'Allemagne, en raison de leur contexte politique et culturel : l'Allemagne obéit à un découpage en Länder, et la Belgique respecte un découpage linguistique, dépendant de deux organismes publics différents, la RTBF (francophone) et la VRT (flamand).

$\mathrm{Au}$ sortir de la Seconde Guerre mondiale, une telle configuration se construit progressivement et le principe du service public se façonne soit en fonction de l'histoire des pays, soit en fonction de ce que les autres pays décident en termes de politique de programmation, de financement, etc.

\section{La création des chaînes privées en Europe}

La télévision de service public exerce plus de poids au fur et à mesure des années, et même si elle tente de faire jouer la complémentarité entre les chaînes pour toucher un public plus large et offrir une programmation plus riche, elle devient rapidement l'objet de vives critiques. Ses principaux opposants viennent de différents milieux, citoyens, associatifs ou encore industriels. Les deux premiers émettent de vives critiques au sujet du monopole dont la télévision de service public jouit, de la collusion entre les hommes politiques au pouvoir et l'information télévisée, ou encore du lien trop étroit entre les hommes politiques et les responsables nommés à des postes clés de la communication audiovisuelle. Les derniers (les 
industriels) revendiquent de plus en plus fortement leur présence dans le champ télévisuel, car ils y ont bien évalué tout l'intérêt qu'une chaîne privée peut leur apporter.

R. Chaniac (Chaniac, op. cit.) identifie trois types de raison à l'origine des changements impliqués par la télévision commerciale et de la remise en cause de la télévision publique : des raisons d'ordre économique, technique ou encore politique. Principalement en raison du coût exigé par la création de nouvelles chaînes ou encore par l'augmentation du temps accordé à la diffusion télévisuelle, la nécessité de mettre en place un financement mixte s'impose dans plusieurs pays. L'apport assuré par la publicité en plus de la redevance améliore la situation en termes financiers, mais demande alors à considérer autrement le principe de la télévision de service public. Ce constat est commun au niveau européen à la fin des années 60. Même si le financement privé s'avère très règlementé et minime, comparativement au financement des chaînes privées, il remet en cause les principes qui président à la télévision de service public. Progressivement, la mixité du financement ouvre la porte à un discours libéral prônant la nécessité de la libre concurrence et du système privé comme garants de pluralisme et de progrès (Chaniac, op. cit.). Ces raisons financières se doublent de raisons techniques, avec l'arrivée du câble et du satellite, multipliant ainsi le choix des programmes, l'accès à de nombreuses chaînes et l'installation à long terme de la télévision commerciale. La raison d'ordre politique concerne essentiellement les pays comme la France et l'Italie, où le pouvoir en place exerce une forte pression, surtout sur les programmes d'informations. Ce contrôle politique ne peut que remettre en cause la légitimité de la télévision de service public, ce que ne manquent pas de contester de nombreux groupes (des associations, les citoyens...) via les radios libres ou des télévisions locales.

Le tableau ci-dessous synthétise les informations en dressant la liste des premières chaînes privées autorisées à diffuser dans l'Europe des douze. Certaines d'entre elles diffusaient déjà avant cette date, mais en toute illégalité. Afin de nous baser sur un critère commun à toutes ces chaînes, nous ne prenons en considération que la date à laquelle, officiellement et après autorisation, elles commencent à diffuser leurs programmes. Pour ce tableau, nous avons choisi de mentionner les douze pays, sans nous limiter cette fois aux cinq étudiés dans les précédentes parties. L'objectif vise à considérer le paysage télévisuel européen dans sa totalité pour mesurer l'arrivée des chaînes privées et son échelonnement en fonction des pays. Classées de la plus ancienne à la plus récente, elles donnent une idée de la période à laquelle elles ont commencé à concurrencer les chaînes publiques.

Fig. 2 : Année de naissance des premières chaînes privées (de la plus ancienne à la plus récente)

\begin{tabular}{|l|l|l}
\hline PAYS & ANNÉE & NoM DE LA \\
\hline ROYAUME-UNI & & CHAÎNE PRIVÉE \\
\hline ITALIE & 1954 & ITV \\
\hline FRANCE & 1971 & Telebiella \\
\hline ALLEMAGNE & 1984 & Canal+ \\
\hline BELGIQUE & 1985 & SAT 1 \\
\hline DANEMARK & 1987 & RTL-TVI \\
\hline ESPAGNE & 1988 & TV2 \\
\hline
\end{tabular}




\begin{tabular}{|l|l|l}
\hline GRECE & 1989 & Mega-Channel \\
\hline PAYS-BAS & 1989 & RTL Véronique \\
\hline LUXEMBOURG & 1991 & RTL \\
\hline PORTUGAL & 1992 & SIC \\
\hline IRLANDE & 1998 & TV3 Ireland \\
\hline
\end{tabular}

Le paysage audiovisuel européen se trouve dans sa grande majorité dans la même configuration : le monopole du service public est remis en cause pour des raisons différentes en fonction des pays, le principe de mixité est adopté et pratiqué. Quelle que soit la manière dont la notion de service public est déclinée dans les différents pays, il apparaît, dès lors que les chaînes publiques rivalisent avec des chaînes privées, que les objectifs inscrits dans leurs cahiers des charges se trouvent logiquement remis en cause. Il ne paraît pas possible de penser atteindre un objectif d'information et d'éducation en occultant sciemment toutes les ressources mises en œuvre par les chaînes privées pour toucher le public le plus large possible, dans une grille de programmation incluant peut-être plus de choix et plus d'intérêt pour les téléspectateurs. Pour la majorité des chaînes de service public, le critère de l'audimat prend plus d'importance encore, de même que celui du financement par la publicité. Les groupes industriels revendiquaient depuis longtemps l'accès plus large à la télévision pour y diffuser leurs publicités et toucher une quantité plus importante de consommateurs par le média de masse représenté par la télévision. Les années 70-80 leur ont permis de mettre en pratique leurs projets, principalement en raison de la crise économique et leurs principes ont souvent été partagés par les hommes politiques au pouvoir avec l'argument fort du principe de liberté de communication (Regourd, op. cit.). De grands groupes industriels font ainsi leur entrée dans le marché de l'audiovisuel et l'on constate que les deux groupes en la matière représentent des poids lourds dans le monde de l'industrie, avec Berlusconi en Italie et Bouygues en France.

Dans les années 80, la télévision française de service public pâtit grandement de la libéralisation de l'audiovisuel avec la privatisation de TF1, l'arrivée de Canal+ et de la Cinq car à elles trois, elles s'accaparent les deux tiers de l'audimat. TF1, à l'origine principale chaîne de service public en termes de parts d'audience et de parts de marché, a été revendue. Elle emporte ainsi et conserve avec elle un capital certain, doublé d'un audimat important. L'arrivée des chaînes privées en Europe a été envisagée dans une logique de « complément » aux chaînes publiques, alors qu'en France elle a été envisagée comme une « substitution » à des chaînes publiques, le cas de TF1 en étant un exemple significatif(Regourd, op. cit.). Canal+, quant à elle, se définit comme la première chaîne privée à péage en France.

Les chaînes publiques, contrairement à l'Italie, ne peuvent renverser la tendance ni retrouver leur place en raison de leurs manques de moyens. L'Italie apparaît cependant comme l'un des rares pays où le secteur public, battu d'abord en brèche par l'arrivée des chaînes commerciales, reprend le dessus pour parvenir à une situation très honorable face à la concurrence. La stratégie insufflée par les responsables de la RAI obéit à la volonté de mettre en commun les éléments propres à chaque chaîne publique pour ensuite miser sur leur complémentarité face aux chaînes privées. Ces éléments se caractérisent par la « coordination des programmes, des ressources, 
des moyens et des achats $»^{2}$. L'Allemagne et le Royaume-Uni, quant à eux, redressent la barre grâce à un financement mieux engagé et à des investissements mieux placés, notamment en production (Chaniac, op.cit.). Ces moyens permettent aux deux pays de mieux supporter la concurrence des chaînes commerciales.

Les changements effectifs et visibles dans la grille de programme impliqués par l'arrivée des chaînes privées se traduisent sensiblement de la même manière en Europe, à quelques différences près. On note avant tout un report des émissions à caractère culturel dans la programmation. Elles sont alors diffusées en première partie de soirée ou beaucoup plus tard. Les émissions de niveau régional perdent du terrain, les chaînes publiques accordent plus de place aux émissions de divertissement (jeux et fictions) et vont désormais sur le terrain des reality shows. Un autre changement se réfère à la régularisation des grilles : l'instauration d'une programmation identique d'un jour à l'autre, permettant de fidéliser l'audience et de rassurer les investisseurs sur des résultats d'écoute sensiblement réguliers, et donc, potentiellement intéressants. Cette technique s'avère d'autant plus efficace qu'elle peut se doubler du principe de « sérialisation »(Chaniac, op.cit), au sens où un programme peut être suivi sur le même créneau sur un temps relativement long.

À la fin de la Seconde Guerre mondiale, les Alliés ont repris en main le système de radiodiffusion allemand dans le but de renverser l'utilisation qui en avait été faite jusqu'alors, à savoir le moyen d'expression d'une pensée unique, selon une conception propagandiste. La reprise en main visait à mettre en œuvre le pluralisme (Regourd, op.cit).

$\mathrm{Au}$ fur et à mesure de son évolution, la question du monopole de la télévision de service public est souvent accompagnée, dans les études consultées, par une distance des instances télévisuelles plus ou moins efficace vis-à-vis du pouvoir politique. Cette distance s'avère toute relative en raison, encore une fois, de l'importance et de l'intérêt stratégique représentés par la télévision aux yeux des hommes au pouvoir. La franche scission entre ce média et le pouvoir paraît cependant complètement utopique (Regourd, op.cit) car le poids exercé par le pouvoir sur la télévision a surtout été visible dans les principes qui ont présidé au règlement et à la composition de la télévision, dans la programmation, mais aussi dans la diffusion des images. Le paysage audiovisuel européen obéit ainsi pour une grande part à la même reconfiguration, passant du monopole du service public à l'arrivée de chaînes privées, pour des raisons différentes en fonction des pays. Que ce soit en termes de « substitution » ou de « complémentarité » entre les chaînes de service public et les chaînes privées, la mixité du financement audiovisuel va donner essor à un nouveau type de programmation. 


\section{$R \cdot E \cdot F \cdot E ́ R \cdot E \cdot N \cdot C \cdot E \cdot S \quad N \cdot O \cdot T \cdot E \cdot S$}

ALDRIDGE, M., MAZDON, L., « La télévision britannique : un enjeu public », in Les lucarnes de l'Europe, Télévisions, cultures, identités, 1945-2005, Paris, Publications de la Sorbonne, 2008, pp.115-124.

BOURDON, J, Histoire de la télévision française sous de Gaulle, Paris, 1994, INA-La Documentation française.

BOURGEOIS, I., Radio et télévision publique en Allemagne. Un modèle à l'épreuve de la nouvelle Europe, 1993, Paris, CIRAC.

CHANIAC, R., «Télévisions publiques en Europe : crise et mutations », in Les lucarnes de l'Europe, Télévisions, cultures, identités, $1945-$ 2005, Paris, Publications de la Sorbonne, 2008, pp.151-167.

DAGNAUD, M., L'État et les médias. Fin de partie, Paris, 2000, éd. Odile Jacob.

DEFRANCE, C., « La reconstruction de la télévision en République fédérale d'Allemagne et la consolidation de l'identité nationale ", in Les lucarnes de l'Europe, Télévisions, cultures, identités, 1945-2005, Paris, Publications de la Sorbonne, 2008, pp.125-138.

JEANNENEY, J.N., Une histoire des médias. Des origines à nos jours, Paris, 1996, Ed. du Seuil.

LITS, M., « La Belgique ou l'illusion de la cohabitation », Hermès 51, 2008, pp. 166-171.

REGOURD, S., La télévision des Européens, Paris, 1992, La documentation française.
1. Chaniac, op.cit.: 159.

2. Chaniac, $2008: 162$ 


\title{
$R \cdot E ́ \cdot S \cdot U \cdot M \cdot E ́$
}

Après la Seconde Guerre mondiale, le projet de mettre en œuvre une action culturelle européenne grandit afin de bâtir l'Europe sur des consolidations solides. Une littérature conséquente en Sciences de l'information et de la communication, en Histoire, en Sociologie a rendu compte des évolutions des télévisions européennes. Nous nous sommes appuyé sur certains des ces travaux pour mener une étude comparative entre quelques pays et analyser les résultats qui traduisent les tendances politiques alors en œuvre dans la mise en place des chaînes publiques et privées. Ces résultats permettent ensuite de mieux évaluer la place occupée par la France dans la politique de service public/privé qu'elle choisit de mener.

\begin{abstract}
After the Second World War, the project to implement a European cultural activity grows in order to build Europe on solid bases. A consequent literature deals with the evolutions of European televisions. This article compares some European countries to analyze the results which then translate the political tendencies into work for public and private channels.
\end{abstract}

